\title{
Determinantes da organização da atenção em diabetes mellitus na satisfação do usuário
}

\section{Determinants of the organization of diabetes mellitus care in user satisfaction}

Fabiana CM Zacharias" ${ }^{1}$; Mariana FS Gomide²; Tânia SG Carneiro²; Alba LS Pinheiro³; Alexandre F Bulgarelli4; Isabela V Lemos5; Brisa PG Figueira5; Ricardo A Arcêncio6; Ione C Pinto7

\begin{abstract}
RESUMO
O objetivo do estudo foi analisar os determinantes da satisfação do usuário com Diabetes Mellitus (DM) sobre o atendimento no ambulatório de especialidades de um Centro de Saúde Escola. O estudo transversal com abordagem qualitativa foi desenvolvido no ambulatório de especialidades com 20 usuários com diagnóstico de DM. Foram aplicadas entrevistas semiestruturadas por meio de um roteiro norteador. Utilizou-se software Atlas ti 7.0 para auxílio na codificação das falas e técnica de Análise de Conteúdo para análise dos dados. Como resultado, observou-se que os temas mais evidenciados estão relacionados à acessibilidade organizacional e à relação entre usuário, equipe e serviço na perspectiva da longitudinalidade. Observou-se a identificação do ambulatório de especialidade como fonte regular de cuidado e a insatisfação no que se refere à acessibilidade ao serviço.
\end{abstract}

Palavras-chave: Avaliação de Serviços de Saúde. Diabetes mellitus. Satisfação do usuário.

\section{Introdução}

O diabetes mellitus (DM) compõe rol de agravos prioritários na área da saúde no Brasil que tem consumido parte dos recursos públicos sanitários pelo aumento das internações em decorrência do agravamento da doença e por levar a mortes prematuras no país. ${ }^{1}$
Estudos epidemiológicos revelam o significativo aumento da prevalência de DM, todavia a maioria dos sistemas de saúde não tem acompanhado essa tendência, revelando-se frágeis para lidar com o problema. Há consenso entre pesquisadores sobre a necessidade de desenvolver novas tecnologias e melhorar as já existentes para prevenção de diabetes, detectá-lo precocemente e evitar danos. Destaca-se
1. Enfermeira, mestre em Ciências pelo Programa de Enfermagem em Saúde Pública da Escola de Enfermagem de Ribeirão Preto da Universidade de São Paulo (EERP-USP); 2. Enfermeiras, mestres, doutorandas, Ciências pelo Programa de Enfermagem em Saúde Pública da EERP-USP; 3. Docente, Universidade Estadual de Santa Cruz, mestre em enfermagem, doutoranda em Enfermagem em Saúde Pública pela EERP-USP. 4. Departamento de Odontologia Preventiva e Social da Faculdade de Odontologia da Universidade Federal do Rio Grande do Sul (FO-UFRGS); 5. Graduandas do Curso de Bacharelado em Enfermagem - EERPUSP; 6. Docentes, Departamento de Enfermagem Materno Infantil e Saúde Pública - EERP-USP.
Correspondência:

Fabiana Costa Machado Zacharias

Avenida dos Bandeirantes, 3900 Campus Universitário - Bairro Monte Alegre Ribeirão Preto - SP - Brasi

CEP: $14040-902$

Artigo recebido em 6/08/2013 Aprovado para publicação em 05/02/2014 
ainda a necessidade de reorganizar os recursos para melhorar a eficácia dos cuidados de saúde no Brasil. ${ }^{2}$

Com o propósito de contribuir para a redução da morbimortalidade associada ao DM no Brasil, o Ministério da Saúde (MS) elaborou o Plano de Reorganização da Atenção à Hipertensão Arterial e Diabetes Mellitus (PRAHD), estabelecendo como metas o aumento da prevenção, detecção, tratamento e controle de agravos no âmbito da atenção básica. ${ }^{3}$

Para tal, urge investimentos a Atenção Primária à Saúde (APS) para a garantia do diagnóstico e favorecimento da vinculação do usuário às unidades de saúde, para tratamento e acompanhamento, promovendo a reestruturação e a ampliação do atendimento resolutivo e de qualidade aos usuários com diabetes. ${ }^{3}$

No âmbito municipal, a Secretaria Municipal de Saúde, desde 2006, com o Programa de Doenças Crônicas Degenerativas, no contexto dos programas de Saúde do Adulto elaborou um Protocolo de Atendimento em Hipertensão e Diabetes com o objetivo de sistematizar as ações em saúde, com vistas à melhoria da qualidade de vida do usuário e, no âmbito da gestão, reduzir gastos com hospitalização e procedimentos de alta tecnologia. ${ }^{4}$

A avaliação dos serviços de saúde tem sido tema constante de discussão no âmbito da academia e dos serviços de saúde e nestes espaços tem-se cobrado a responsabilidade do Estado em garantir estes serviços. Assim, neste sentido cada vez mais se discute a importância de estudos de avaliação como ferramenta ou instrumento para o fortalecimento dos sistemas e serviços de saúde.

A avaliação no setor saúde não se restringe à verificação do cumprimento de objetivos propostos, pelo sistema de saúde, mas também para verificar se esses estão socialmente definidos e se estão de acordo com as aspirações e expectativas dos técnicos da área e dos seus usuários. ${ }^{5}$

A avaliação dos serviços de saúde constitui uma ferramenta de gestão e planejamento da assistência prestada aos usuários. ${ }^{6}$ Portanto, entende-se que trazer a opinião de quem é cuidado e das características do cuidado prestado pode ser uma ferramenta para garantir a qualidade da atenção e portanto receber boa avaliação.

O resultado é definido como seriam as mudanças verificadas no estado de saúde dos usuários que pudessem ser atribuídas a um cuidado prévio. ${ }^{7}$ Dentre os indicadores de avaliação de serviços de saúde proposta pela Sociedade Brasileira de Diabetes em 2009, está a satisfação do usuário.

A satisfação do usuário é de fundamental importância como medida da qualidade da atenção, porque proporciona informações sobre o êxito do provedor em alcançar os valores e expectativas dos usuários. ${ }^{8}$ É importante dar voz ao usuário, expressando suas expectativas quanto ao uso do serviço, favorecendo, assim, um diálogo para a tomada de decisão, na direção de serviços voltados para atender as necessidades dos usuários. ${ }^{9}$

As atitudes do usuário, como, por exemplo, sua satisfação, o conhecimento adquirido sobre a sua saúde e a transformação do seu comportamento, relacionado à sua saúde, poderiam ser considerados como resultados na avaliação. ${ }^{10}$

Conforme observado na literatura, estudos envolvendo a satisfação do usuário trazem com grande frequência a utilização de entrevistas com o método de análise de conteúdo na modalidade temática como ferramenta para avaliar o processo de acolhimento, bem como a satisfação com o atendimento recebido. ${ }^{9,11,12,13}$

Assim, o estudo tem como objetivo analisar os determinantes da satisfação do usuário com DM em relação ao atendimento no ambulatório de especialidades de um Centro de Saúde Escola.

\section{Material e Métodos}

Estudo transversal com abordagem qualitativa, realizado em um ambulatório de especialidades, mais especificamente ambulatório de endocrinologia e ambulatório integrado de um serviço referência para atenção em DM, que funciona de $2^{\mathrm{a}}$ a $6^{\mathrm{a}}$ feira prestando assistência das 7 às $20 \mathrm{~h}$ mediante agendamento de consultas.

A dinâmica do serviço conta com atendimento médico realizado por alunos do $5^{\circ}$ ano de graduação de medicina, com supervisão de docentes, Ambulatório Integrado. Essa assistência ambulatorial é parte de estágio que objetiva atuar na assistência integral contínua, visando boa qualidade no atendimento às necessidades de saúde da população ${ }^{14}$ e alunos do $4^{\circ}$ ano de enfermagem em estágio supervisionado que objetiva desenvolver conhecimentos, habilidades e atitudes na área de competência do cuidado. ${ }^{15}$

Os usuários são atendidos por meio de referência das Unidades Básicas de Saúde (UBS) do distrito e de demandas do ambulatório de especialidades. 
Usuários que necessitam de atendimento especializado de áreas não constantes neste serviço são encaminhados para outros níveis de assistência, ambulatoriais e hospitalares, que compõem o SUS.

Os sujeitos deste estudo são os usuários com diagnóstico de DM, confirmados em prontuários de saúde, adultos e idosos, de ambos os sexos. Foram selecionados de forma intencional usuários maiores de 18 anos, com diabetes mellitus tipo 2 (há 2 anos), em seguimento no ambulatório de endocrinologia e/ou integrado do local do estudo há pelo menos um ano e morar há mais de um ano na área de abrangência do distrito. Os critérios de exclusão dos sujeitos foram: apresentar sinais e/ou sintomas de confusão mental ou embriaguez.

Realizou-se imersão ao campo de investigação e, pela técnica da observação simples, utilizando o espaço físico em que os usuários aguardavam para consulta médica. Desses, foram sorteados, no momento da visita da pesquisadora ao referido local da pesquisa, de forma aleatória, 20 usuários para serem entrevistados, sendo entrevistado um usuário por período de coleta, no referido ambulatório. O número de sujeitos participantes parte do pressuposto de ser uma amostra intencional suficiente para trabalhar o referido objeto de pesquisa por meio de uma pesquisa de desenho metodológico qualitativo. Vale ressaltar a observância da repetição dos dados sendo também critério para interrupção das entrevistas.

O sorteio foi realizado diariamente, por um período de 3 meses $\left(1^{\circ}\right.$ de agosto a 30 de outubro de 2012), entre os dias de segunda a sexta-feira durante o horário de atendimento ambulatorial, mediante a lista de atendimento, sendo escolhido dentre os usuários aqueles que estivessem no serviço aguardando para consultas médicas.

Os dados foram coletados a partir de um roteiro norteador, por meio de entrevista semiestruturada, conduzida com base numa estrutura solta, com questões abertas que definiram a área a ser explorada, a partir da qual o entrevistador, ou o entrevistado, pode divergir, a fim de prosseguir com uma ideia ou uma resposta em maiores detalhes. ${ }^{16}$

Foram abordadas questões relativas à satisfação do usuário em relação ao local de atendimento e sua adequação, a realização de exames laboratoriais e acesso às consultas, bem como sua percepção quanto ao fluxo de atendimento pela equipe de saúde e acolhimento recebido.

As entrevistas foram realizadas em consultóri- os médicos, disponíveis no serviço de saúde, a fim de garantir privacidade e o mínimo de desconforto durante a conversa.

Após transcrever as entrevistas, os dados foram gerados por meio de um banco de dados com o uso do software Atlas ti, versão 7.0, mediante a criação da Hermeneutic Unit, em que foram inseridas as 20 entrevistas. Posteriormente, a minuciosa leitura das entrevistas, foi realizada a codificação do material empírico, das entrevistas, de modo a apreender os elementos que se integravam ao objeto do estudo, para tal foram criados 12 códigos (vínculo com equipe de enfermagem; vínculo com médico; tempo de espera para consulta; agendamento de consultas e retornos; acesso a medicamentos; acesso a realização de exames laboratoriais; acesso a resultados de exames laboratoriais; cadastramento do usuário nos sistemas).

Após a elaboração dos códigos, foram abertas uma a uma as entrevistas sendo codificadas por meio de sublinhamento dos fragmentos, podendo esses receberem mais de um código. Ao final da codificação do material, foram gerados relatórios constando separadamente os fragmentos correspondentes a cada código com a sinalização da entrevista correlativa.

$\mathrm{Na}$ análise dos dados foi adotada a técnica da Análise de Conteúdo, modalidade temática. ${ }^{17}$ Todo material coletado foi lido exaustivamente e discutido, com apreciação das falas fazendo aproximações de ideias e marcação dos fragmentos de respostas, localizando as ideias centrais. Desta forma, o objeto da presente pesquisa foi analisado por meio da técnica de Análise de Conteúdo pois a mesma firma-se como um conjunto de técnicas de análise das comunicações em que palavra é levada em consideração para compreensão dos seus significados. ${ }^{17}$ As diferentes fases da análise de conteúdo se organizaram em torno de três polos cronológicos: a pré-análise, a exploração do material e o tratamento dos resultados, a interferência e a interpretação. ${ }^{17}$

Assim, para a análise temática, foram seguidas três etapas divididas em organização dos dados, considerando os objetivos da pesquisa e organizando o material, de forma a responder as questões de validade quanto à exaustividade, representatividade, homogeneidade e pertinência; exploração do material empírico, com leitura exaustiva dos registros, codificação dos dados, separação dos fragmentos de acordo com as convergências, divergências e o que fosse inusitado e a classificação e agregação dos dados em temas. 
O estudo foi submetido e aprovado pelo Comitê de Ética e Pesquisa (CEP) com CAAE: 02446112.9.0000.5393, atendendo as Diretrizes e Normas Regulamentadoras de Pesquisas.

\section{Resultados}

$\mathrm{Na}$ organização das falas o material empírico codificado possibilitou observar que os temas mais evidenciados estão relacionados à acessibilidade organizacional e à relação entre usuário, equipe e serviço na perspectiva da longitudinalidade. Assim, têm-se duas categorias temáticas intituladas: 1- Acessibilidade organizacional - a percepção do usuário e 2- O cuidado ao usuário com DM - a longitudinalidade em foco. A seguir, as categorias temáticas serão apresentadas, com exemplos extraídos das falas, que serão identificados com a letra $\mathrm{U}$, correspondente ao usuário, seguida dos números de 1 a 20 , sequencialmente.

Dentre os 20 usuários entrevistados, $30 \%$ eram do sexo masculino e $70 \%$ do sexo feminino. A idade variou de 43 a 80 anos.

\section{Categoria temática 1: A acessibilidade orga- nizacional - a percepção do usuário}

Considerou-se acessibilidade organizacional como representada pelos obstáculos nos modos de organização dos recursos de assistência à saúde. Tais obstáculos podem se apresentar desde a entrada no serviço de saúde, como a demora em obter uma consulta, tipo de marcação de horário e turnos de funcionamento, inclusive pode estar no interior da unidade de saúde como o tempo de espera para ser atendido pelo médico e para fazer os exames laboratoriais. ${ }^{18}$ As falas seguintes relatam a organização do serviço, no que tange o agendamento para retorno de consultas.

[...] eu venho ele [médico] já dá um papel com dia para gente fazer o exame com as orientações e já marcam o retorno, não preciso vir aqui pra marcar o retorno [...](U10).

[...] por exemplo, saí hoje e já fica marcado o retorno para o próximo, só se acontecer que não puder atender eles me ligam [...] (U17).

[...] você faz todo os exames, constata que está doente, apesar do sistema precário, com muito sacrificio esperando 2, 3, 4, 5 e meses a passar por um médico especialista leva mais 2, 3 meses para fazer exames e retornar a este médico, leva às vezes 2 a 3 anos para descobrir uma doença grave (U9).
[...] para marcar consulta só que é difícil, até que abre a agenda, a gente que é paciente diabético às vezes nesse intervalo está passando mal, está descompensado, a gente não sabe o que fazer, e você vai ser atendido só na outra consulta em seis meses, aí eu acho que tinha que ter uma maneira de ser atendida fora da época de uma consulta dessa (U2).

[...] a única coisa é que demora nas consultas... que é de 4 meses, às vezes nesse intervalo, porque minha diabetes cai muito, ultimamente ela está caindo muito então eu tenho que esperar e controlar em casa, ou então esperar o retorno [...] (U18).

Eu passo mesmo só com o médico, quando eles marcam meu retorno é a cada 3 meses, mas agora dessa vez marcaram pra quatro eu não sei por que, até vou falar com o médico porque marcaram para quatro meses e depois a receita vence e aí eu fico assim. É um sufoco para pegar a receita (U5).

Quando não se faz o agendamento na sala de pós-consulta], fica difícil depois de você fazer porque não pode ser feito por telefone. Eu que moro em um bairro tenho que ficar vindo para ver se abriu agendamento [...] (U12).

Foram identificadas outras falas que também relataram sobre a organização do serviço quanto ao fluxo interno de atendimento pelos profissionais.

Primeiro passa para pesar, esses negócios... depois aguarda eles chamarem... depois tem a médica, a residente... depois ela passa por mim e vai conversar com a chefe dele, às vezes ela vem aqui encher a minha cabeça, às vezes não vêm... aí vai na sala de pós-consulta], eu estou cheio disso aqui (U15).

A médica passa para outro profissional e ele me orienta que é a enfermeira [enfermagem] (U8).

As falas que seguem expressam a espera no serviço desde a chegada do usuário até o atendimento profissional para consulta ou realização de exames.

É igual te falei eu acho muito deficiente, acho que tinha que melhorar e muito, pois ficamos mais tempo esperando do que para ser atendido mesmo [...] a gente espera muito tempo, eles ficam lá três quatro dentro da sala batendo papo e vinte pessoas lá de fora para serem atendidas (U7). 
É que demora um pouquinho, como a senhora viu, até que a gente vai para consultar mesmo demora (U8).

[...] até que eles vêm, você fica ali oh, meia hora, mais de meia hora esperando, só isso aí só que eu acho ruim (U5).

Ah, como eu só venho só no retorno [...] quando tem muita gente, às vezes tem que até ficar em pé esperando [...] eu como sempre venho em horário marcado então não tenho esse problema (U19).

[...] eu acho um pouco demorado para coletar (exames), quando eu chego é muita gente né, muita gente colhendo (U4).

Demora muito os exames (U14).

Constatou-se uma fala que relatou o horário de funcionamento do Ambulatório de Especialidades de Saúde.

[...] tem a nutricionista aqui inclusive ela já me convidou, mas eu não posso eu trabalho, não tem como, assim as pessoas me ligam pra mim está vindo, mas não tem como eu vir toda semana aqui porque a gente trabalha, a gente conseguiu um tempo pra vir na consulta, pra explicar que não dá tempo (U3).

\section{Categoria Temática 2: 0 cuidado ao usuário com DM - a longitudinalidade em foco}

Esta categoria fundamentou-se no conceito de longitudinalidade definido como uma relação pessoal, de longa duração, entre os profissionais de saúde e os usuários em suas unidades de saúde, no contexto da atenção primária ${ }^{19}$. Nesse sentido, entende-se que, para este trabalho, tal atributo pode estar presente no ambulatório de especialidade para o cuidado de usuários com DM, visto que a recomendação pelo MS é que pacientes com DM de difícil controle e/ou presença de comorbidades, usuários de insulina e diabetes gestacional devem ser acompanhados por especialistas em nível de atenção mais complexo ${ }^{20}$, o que demanda seguimento prolongado nesses serviços.

As falas a seguir apontam a fragilidade do vínculo terapêutico ao ser prestado por diferentes profissionais a cada atendimento.

Eu só faço acompanhamento do médico aqui e do curativo... os médicos são difíceis porque cada retorno é um que atende [...] São difíceis os médicos repetirem, se for para te falar até o nome de quem me atendeu por último eu não sei (U17).

Eu tratava com o doutor $X$, o doutor $X$ saiu, aí cada dia que você vem é um médico que atende, então eu não sei não (U2).

Cada vez que vem é um que atende a gente (U14).

Nem sempre é o mesmo aluno [de medicina] que vai atender, você acaba não criando vínculo com o médico [...] Ai você não cria muito vínculo porque cada vez que você vem é um (U12).

Cada dia que venho aqui é uma pessoa diferente... a gente gostaria de falar diretamente com o médico, que é muita coisa que você quer dialogar com ele, aí a gente não se sente a vontade de falar com os estagiários [...] Então quem atende é os estagiários faz muito tempo que não vem médicos diretamente como vinha (U8).

As falas mostram a identificação do ambulatório de especialidades como fonte regular de cuidado.

[...] 26... 27 anos que faço tratamento aqui... para mim é normal o tratamento aqui (U 16).

[...] nunca fui mal atendido, e não é uma nem duas vezes que eu venho, que eu venho direto... tanto médico, como curativo... então eu não tenho que reclamar de nada... eu sempre fui muito bem atendido (U17).

Eu acho que aqui eu tenho mais ou menos, eu penso, preciso ver na pasta já tem uns 15 anos aqui até mais, então toda vida eu fui muito bem atendida (U16).

Faz alguns anos que venho aqui e é legal. Não tenho nenhuma dificuldade de comunicar em situações específicas que eu fico com alguma dúvida entre os retornos eu venho e converso (U11).

\section{Discussão}

No que se refere à satisfação do usuário com DM em relação ao atendimento no ambulatório de especialidades, abordou-se a expectativa do usuário nas dimensões satisfação e insatisfação.

Quanto à acessibilidade organizacional, os resultados apontam para a organização do serviço no que tange ao agendamento para retorno de consultas; e fluxo interno de atendimento pelos profissionais; também esteve evidenciada a espera no serviço desde a chegada do usuário até o atendimento profissional para consul- 
ta ou realização de exames; ao horário de funcionamento do Ambulatório de Especialidades de Saúde. ${ }^{18}$

Sobre o item organização do serviço, no que se refere ao agendamento para retorno de consultas percebeu-se que os usuários em maior frequência relatavam insatisfação nesse quesito, corroborando com outro estudo realizado na APS, em que os resultados apontam que a grande maioria dos usuários tem expectativas que a consulta tivesse menor tempo para o agendamento ${ }^{10}$. Em outro estudo no mesmo município sobre acessibilidade e demanda em Unidade de Pronto Atendimento, os usuários relatam a demora pelo atendimento e agendamento de consultas na atenção básica justificando a procura desse serviço para o ingresso aos serviços de saúde. ${ }^{12}$

Quanto ao fluxo interno de atendimento pelos profissionais e a espera no serviço para consulta ou realização de exames, foi identificado, no estudo que os usuários permanecem considerável tempo aguardando pelo atendimento no corredor do serviço, expressando insatisfação. Estes achados corroboram outro estudo realizado na APS. ${ }^{9}$ Tais resultados demonstram a problemática em diferentes pontos de atenção no município e traz reflexão quanto à organização destes serviços para além da prática médica, que pudessem instituir ações desenvolvidas por equipes multiprofissionais. Tal investimento desconcentraria consideravelmente as demandas por consulta médica ao mínimo, impactaria a qualidade da atenção. Os locais que conseguiram instituir um trabalho organizado por equipes multiprofissionais estão produzindo mais satisfação de seus usuários e resolutividade em seus processos, sendo isto mais auto-efetivo.

Há que destacar a própria ambiência que se estabelece de cooperação entre atores e reconhecimento de sua interdependência e autonomia. Em que prezem todas as ações e avanços do serviço investigado no atendimento à clientela, ainda a prática está centrada em trabalhos individuas que por vezes se sobrepõe e até são redundantes, como relatado pelo usuário ter que responder à equipe de enfermagem e depois à médica.

Quanto à insatisfação sobre o horário de funcionamento do ambulatório de especialidades de saúde, um dos relatos traz o descompasso entre o horário de funcionamento e a necessidade do usuário representando uma barreira à acessibilidade organizacional. ${ }^{13}$

No que se refere à longitudinalidade no cuidado ao usuário com DM, os achados do estudo corroboram as dimensões apresentadas para o contexto do
Sistema Único de Saúde, ao apresentarem a identificação da unidade básica como fonte regular de cuidado, vínculo terapêutico duradouro e continuidade informacional como componentes para esse atributo. ${ }^{21}$

Ao se tratar do acompanhamento do usuário com DM, ao longo do tempo, as falas mostraram a identificação dos ambulatórios de endocrinologia e/ou integrado como fonte regular de cuidado, considerado para este estudo como satisfação.

No entanto, a dinâmica da assistência prestada por diferentes profissionais a cada atendimento (médicos residentes, alunos de graduação de enfermagem e medicina que passam pelo serviço de acordo com a programação de estágio a ser cumprido) apontam a fragilidade do vínculo terapêutico, sinalizando a insatisfação do usuário.

O cuidado longitudinal é favorecido principalmente pelo longo tempo de atuação na mesma unidade de saúde, pressupõe a manutenção de vínculos duradouros com os usuários ${ }^{18}$, no entanto, sobre a relação profissional e usuário há fragilidade visto que os usuários relataram ser atendidos cada vez por um profissional médico diferente.

Entende-se que a existência de uma relação profissional-usuário satisfatória e reconhecida pelos usuários demonstra o grau de responsabilidade do serviço de saúde com a população, e ainda, que essa relação de respeito, compreensão e escuta faz a diferença entre as práticas de saúde. ${ }^{22}$

Quando os profissionais conhecem melhor os usuários, eles são mais capazes de julgar a necessidade de intervenções diagnósticas e de avaliar diferentes modos de intervenção. Essas vantagens são mais bem alcançadas quando a relação é com um profissional em particular e não com um local específico. ${ }^{9}$

Considera-se como limitações do estudo a dificuldade na disponibilização de sala no horário de atendimento ambulatorial para realização das entrevistas, bem como o fato destas terem sido realizadas no serviço podendo influenciar na resposta dos informantes, gerando um viés de informação e memória que podem ter interferido nos resultados.

Além disso, percebeu-se que seria importante também ter ouvido os profissionais acerca do cuidado oferecido no serviço para usuários com DM.

\section{Conclusões}

Os determinantes da organização da atenção em DM foram elencados neste estudo como a acessi- 
bilidade organizacional e a longitudinalidade do cuidado relacionado usuário, equipe e serviço.

Os achados evidenciaram acessibilidade organizacional como obstáculo para a satisfação dos usuários no que se refere ao agendamento para retorno de consultas, ao fluxo interno de atendimento pelos profissionais e a espera no serviço desde a chegada do usuário até $\mathrm{o}$ atendimento profissional para consulta ou realização de exames além do horário de funcionamento da unidade de serviço.

Por se tratar de um Centro de Saúde Escola, a dinâmica de atendimento por diversos profissionais é inerente ao serviço. No entanto, foi observado que há fragilidade na relação profissional e usuário pelo atendimento ser realizado cada vez por um profissional diferente.
O estudo traz informações que servirão de subsídios para gerentes de Unidades de Saúde, bem como gestores do sistema público de saúde na formulação de políticas no âmbito do acolhimento e vínculo das pessoas com essa condição crônica.

Os resultados poderão contribuir para o embasamento de outros conhecimentos na área da avaliação da qualidade de serviços de saúde no SUS, bem como qualificar a própria atenção ofertada a esses usuários, para mais qualidade de vida e bem-estar social.

\section{Agradecimentos}

À Fundação de Amparo a Pesquisa do Estado de São Paulo, FAPESP, pela concessão de bolsa de mestrado à primeira autora, processo 2011/04646-7.

\begin{abstract}
The objective of the study was analyze user satisfaction determinants of patients with Diabetes Mellitus (DM) regarding the outpatient clinic specialties at the Health Centre of the School. Qualitative crosssectional study was carried out in outpatient specialty with 20 users with diagnosis of DM. We adopted the method of semi-structured interview using a scripting guide. We used Atlas it software 7.0 to aid in the decoding of speech, and analyzed it by content analysis. The empirical material encoded enabled to observe that the most evident themes are related to organizational accessibility and the relationship between user and service staff in perspective of longitudinality. It was observed the outpatient identifying clinic specialties as a regular source of care and dissatisfaction with regard to service accessibility.
\end{abstract}

Keywords: Health Services Evaluation. Diabetes mellitus. Consumer Satisfaction.

\section{Referências}

1. Oliveira AF, Valente JG, Leite IC, Schramm JMA, Azevedo ASR, Gadelha AMJ. Global burden of disease attributable to diabetes mellitus in Brazil. Cad Saúde Pública. Rio de Janeiro, 2009. 25: 1234-44.

2. Bahia L, Araujo DV, Schaan BD, Dib SA, Negrato CA, Leão MPS, et al. The costs of type 2 diabetes mellitus outpatient care in the Brazilian Public Health System. Value health. 2011; 14(Supplement 1): S137-40.

3. BRASIL. Ministério da Saúde. Secretaria de Políticas de Saúde. Departamento de Ações Programáticas Estratégicas. Plano de reorganização da atenção à hipertensão arterial e ao diabetes mellitus: hipertensão arterial e diabetes mellitus. Brasília, DF, 2001. (Série C. Projetos, Programas e Relatórios, n. 59).

4. Secretaria Municipal da Saúde (SP). Protocolo de atendimento em hipertensão e diabetes. Ribeirão Preto (SP): Prefeitura Municipal. Secretaria Municipal da Saúde; 2006.

5. Donabedian A. Evaluación de la calidaded de la atención medica. New York, Milbank Q. 1966; 44: 383-404.
6. Silva ASB, Santos MA, Teixeira CRS, Damasceno MMC, Camilo $\mathrm{J}$, Zanett ML. Avaliação da atenção em diabetes mellitus em uma unidade básica distrital de saúde. Florianópolis, Texto \& contexto enferm. 2011; 20: 512-8.

7. Donabedian A. Exploration in quality assessment and monitoring. Ann Arbor: Health Administration Press; 1980. Basic approaches do assessment: structure, process, outcome; p.77-125.

8. Vaitsman J, Andrade GRVB. Satisfação e responsividade: formas de medir a qualidade e a humanização da assistência à saúde. Ciênc Saúde Coletiva. 2005; 10:599-613.

9. Gaioso VP, Mishima SM. User satisfaction from the perspective of acceptability in the family health scenario. Texto \& contexto enferm. 2007; 16:617-25.

10. Donabedian $A$. The role of outcomes in quality assessment and assurance. Chicago, QRB Qual Rev Bull. 1992; 18: 35660.

11. Baratieri T, Mandu ENT, Marcon SS. Longitudinalidade no trabalho do enfermeiro: relatos da experiência profissional. Rev Esc Enferm USP 2012; 46:1260-7. 
12. Gomide MFS, Pinto IC, Figueiredo LA. Accessibility and demand at an Emergency Care Unit: the user's perspective. Acta Paul Enferm. 2012; 25(spe2): 19-25.

13. Moimaz SAS, Marques JAM, Saliba O, Garbin CAS, Zina LG, Saliba NA. Satisfação e percepção do usuário do SUS sobre o serviço público de saúde. Physis. (RIO J.) 2010; 20:1419-40.

14. Universidade de São Paulo (BR) [Internet]. São Paulo: Universidade; c1999-2013 [citado 2013 maio 2]. Júpiter - Sistema de Graduação: Faculdade de Medicina de Ribeirão Preto: Disciplina: RCG0510 - Estágio Integrado em Centros de Saúde e Medicina Comunitária. Disponível em: https:// sistemas2.usp.br/jupiterweb/obterDisciplina? sgldis= RCG0510\&nomdis.

15. Universidade de São Paulo (BR) [Internet]. São Paulo: Universidade; c1999-2013 [citado 2013 maio 22]. Júpiter - Sistema de Graduação: Escola de Enfermagem de Ribeirão Preto: Disciplina: 2200083 - Estágio curricular: enfermagem na atenção básica. Disponível em: https://sistemas.usp.br/ jupiterweb/obterDisciplina?sgldis=2200083\&verdis $=4$.

16. Pope C, Ziebland S, Mays N. Analisando dados qualitativos. In: Pope C, Mays N. Pesquisa qualitativa na atenção à saúde. Porto Alegre: Artmed; 2009.
17. Bardin L. Análise de conteúdo. $6^{a}$ Ed. São Paulo: Edições 70 , 2011. 279p.

18. Fekete MC. Estudo da acessibilidade na avaliação dos serviços de saúde. In: Santana JP, organizador. Desenvolvimento gerencial de unidades básicas do Sistema Único de Saúde (SUS). Brasília: Organização Pan-Americana Saúde; 1997. p.114-20.

19. Starfield B. Atenção Primária: equilíbrio entre necessidades de saúde, serviços e tecnologia. Brasília: Ministério da Saúde; 2002.

20. Brasil. Ministério da Saúde. Cadernos de Atenção Básica no 16. Diabetes Mellitus. Brasília; 2006

21. Cunha EM, Giovanella L. Longitudinalidade/continuidade do cuidado: identificando dimensões e variáveis para a avaliação da Atenção Primária no contexto do Sistema Público de Saúde brasileiro. Ciênc Saúde Coletiva. 2011;16 Supl 1:102942.

22. Medeiros FA, Souza GCA, Barbosa AAA, Costa ICC. Aceptación en una Unidad Básica de Salud: enfoque en la satisfacción del usuario. Rev Salud Pública. 2010; 12: 40213. 